\title{
L'étude des représentations sociales de la drogue et du cannabis : un enjeu pour la prévention
}

\section{A study on the social representations of drugs and cannabis: the issues and what is at stake for prevention}

\author{
L. Dany ${ }^{(1,2)}$, T. Apostolidis $(1,3)$
}

Résumé: La drogue et le cannabis sont des objets sociaux complexes et polysémiques. Deux recherches exploratoires à partir d'entretiens semi-directifs $(n=50)$ et de questionnaires $(n=300)$ ont été réalisées afin d'étudier les représentations sociales de ces deux objets, en fonction des caractéristiques sociales des individus (sexe, age) mais aussi de leur proximité au cannabis (consommation versus non consommation). L'étude de ces représentations permet de mettre en évidence la reconstruction sociocognitive que les individus opèrent, reconstruction largement influencée par l'insertion sociale des individus mais aussi par les modalités de consommation de cannabis. La connaissance des représentations apparaît comme un outil de compréhension des réalités souvent complexes liées aux mondes de la drogue et permet de penser des démarches de prévention plus adaptées.

Summary: Drugs and cannabis are two complex, multifaceted social objects. Two exploratory research projects were conducted from semi-directive interviews $(n=50)$ and questionnaires $(n=300)$ in order to investigate the social representations contained in these two objects according to both the social characteristics of individuals (gender, age) and their proximity to cannabis (consumption versus non-consumption). The study of their representations allows for emphasis to be placed upon the socio-cognitive reconstruction that individuals maneuver, reconstruction largely influenced by the social insertion of individuals but also by their method of cannabis consumption. Knowledge of these representations seems to be a tool for understanding the often complex realities linked to the world of drugs and allows for reflection upon more adapted prevention methods.

Mots-clés : représentations sociales - prévention - cannabis - drogue - consommation. Key words: social representations - prevention - cannabis - drugs - consumption.

(1) Laboratoire de psychologie Sociale, Université de Provence, 29, avenue Robert-Schuman, 13621 Aix-en-Provence, France.

(2) Observatoire Régional de la Santé PACA, 23, rue Stanislas-Torrents, 13006 Marseille, France.

(3) Unité INSERM U379, 23, rue Stanislas-Torrents, 13006 Marseille, France.

Tiré à part: L. Dany

Réception: 28/02/2002 - Acceptation: 25/10/2002

ORS PACA

23, rue Stanislas-Torrents

13006 Marseille 


\section{Introduction}

On assiste en France depuis quelques années à la diffusion de nouvelles informations sur les substances psychoactives. Celles-ci viennent se confronter aux représentations préexistantes dans le champ social et entraînent des débats qui s'articulent principalement autour de la dangerosité des différentes substances et des politiques publiques à leur égard (sanitaire, juridique).

\section{La drogue et le cannabis comme objets sociaux}

Son statut permanent dans la vie de I'homme ainsi que le débat qui lui est inhérent font de la drogue un objet complexe, un fait social total [28]. Le concept de drogue apparaît comme non-scientifique, institué à partir d'évaluations morales, portant en son sein la norme et l'interdit [11]. L'imaginaire social joue un rôle fondamental dans la construction du rapport aux drogues et dans la détermination de la place que la société leur attribue [13].

Le cannabis est la substance illicite la plus consommée en France : près de 5 millions de personnes en auraient déjà consommé [22], 40,5\% des 1834 ans l'auraient déjà expérimenté [23]. II existe, par ailleurs, une grande diffusion de sa consommation dans tous les milieux sociaux sans exception [16].

Mais, le cannabis est en France une substance illicite et c'est de la confrontation entre sa banalisation et son statut juridique qu'émerge le débat. Celui-ci n'est pas le seul fait des spécialistes, mais se situe à tous les niveaux de la société. La communication opérée par les médias illustre d'ailleurs parfaitement la perception "plurielle» et le caractère polémique qui prédominent au sujet de cette substance.
Récemment, à l'occasion de la diffusion d'un rapport d'expertise sur les effets de la consommation de cannabis sur la santé, commandé à I'INSERM [17] par la MILDT, certains quotidiens nationaux titraient "Une étude de l'Inserm relativise les dangers de la consommation du cannabis » (4), quand d'autres titraient sur "Les dangers du cannabis reconnus » (5).

\section{La consommation de drogue : une pratique sociale}

Les usages de drogues sont des pratiques éminemment sociales. Les usages déclarés sont régulés par des déterminants sociaux comme l'âge et le sexe. On observe en effet une surconsommation masculine et ce, quelle que soit la substance (à l'exception des médicaments psychotropes), ainsi qu'une consommation de substances illicites plus importante chez les plus jeunes [14, 23]. Le rapport aux substances psychoactives s'inscrit dans un contexte social et culturel porteur de sens : le consommateur (ou le non-consommateur) n'existe pas seul mais en relation avec le système socioculturel dont il est issu [26].

\section{Les représentations sociales comme grilles de lecture}

Par représentations sociales, nous entendons l'ensemble organisé des connaissances, des croyances, des opinions, des images et des attitudes partagées par un groupe à l'égard d'un objet social donné. Étudier les représentations sociales c'est chercher la relation que l'individu entretient au monde et aux choses [18].

Cette optique conceptuelle fournit un modèle pour penser l'articulation entre système de pensée et système

(4) Le Monde du 23 novembre 2001.

(5) Le Figaro du 23 novembre 2001. 
de comportement [2] à partir de l'analyse des dimensions psychologiques, contextuelles et sociales qui rendent compte des relations que les individus développent avec les objets sociaux environnants. L'étude des représentations porte sur les univers interprétatifs (réseaux d'ancrage des informations et des significations attribuées) dans lesquels les objets sont appropriés et légitimés dans les conduites individuelles et sociales. Elle permet de cerner les "grilles de lecture " qui donnent du sens au comportement [19] et constituent de véritables guides pour l'action des individus.

L'intérêt de ce type de recherche réside dans la mise en lumière des processus médiateurs [7] qui régulent le rapport aux objets de notre environnement, dans le cas présent, la drogue et le cannabis. Il s'agit dès lors, non seulement de les identifier, mais aussi d'appréhender leurs dynamiques à l'interface de l'individuel et du collectif. Notamment en ce qui concerne des objets polymorphes comme les substances psychoactives, à la fois porteurs d'enjeux publics (santé publique, cadre légal) et privés (mode de vie).

C'est enfin proposer des pistes de réflexion sur l'impact des actions de prévention [20], qui s'opèrent dans une réalité sociale complexe, que l'étude des représentations sociales permet d'analyser. L'intérêt de ce paradigme repose également sur le fait qu'il permet de mettre en évidence le lien entre représentations et processus intergroupes [34].

\section{Objectifs}

Au regard des données dont on dispose sur le cannabis et son importance; diffusion et banalisation de sa consommation [3, 16], nos objectifs sont :
- d'appréhender les univers représentationnels (signification et image) de la drogue et du cannabis,

- de mettre en relation ces représentations avec les pratiques de consommation de cannabis,

- de comprendre les systèmes de représentations en fonction des appartenances sociales (sexe, âge) des individus.

\section{Méthodes}

Dans le cadre du Laboratoire de Psychologie Sociale de l'université de Provence, nous avons initié un programme de recherche portant sur les conceptions et usages associés aux substances psychoactives. Les résultats suivants sont issus de deux recherches exploratoires effectuées sur Marseille et sa région, le caractère exploratoire de ces recherches est lié au peu d'information dont nous disposons sur le sujet exploré. Une première recherche menée, par entretien semi-directif sur le thème de la drogue, a été réalisée auprès d'une cinquantaine de personnes ${ }^{(6)}$, clivées en fonction de deux variables : le sexe (25 hommes et 25 femmes) et l'âge (25 personnes de 18-25 ans et 25 de 45 ans et plus), le guide d'entretien ${ }^{(7)}$ de cette première recherche comprenait plusieurs thématiques: le thème de la dépendance, les contextes de consommation, le rapport au produit comme facteur explicatif du statut de drogué.

Une seconde recherche sur les représentations de la drogue et du

(6) L'échantillonnage a été réalisé par la technique dite "boule de neige».

(7) La consigne inaugurale visait à cerner les substances spontanément citées comme drogue, ainsi que les thèmes spontanément développés par les individus. 
cannabis, réalisée à partir de questionnaires d'évocations ${ }^{(8)}$, a été conduite auprès d'un échantillon de 300 personnes, clivé en fonction du sexe (150 femmes et 150 hommes) et du type de rapport au cannabis. Ces différentes modalités de rapport au cannabis ont été définies sur la base de la classification proposée par Peretti-Watel [27]. Soit, 100 « abstinents " (personne n'ayant jamais consommé de cannabis), 100 «expérimentateurs " (personne ayant déjà consommé du cannabis, mais pas au cours des douze derniers mois) et 100 consommateurs "réguliers 》 (au moins dix consommations dans les trente derniers jours).

Cet échantillonnage quasi expérimental ${ }^{(9)}$ [10], a été réalisé sur une population «tout venant », volontaire pour participer à cette recherche, appareillée en fonction du sexe ${ }^{(10)}$ et de l'âge ${ }^{(11)}$ pour chaque modalité de rapport au cannabis. Les personnes interrogées étaient âgées de 18 à 35 ans, soit 27,64 ans en moyenne pour les femmes et 26,45 ans en moyenne pour les hommes $(\mathrm{Khl} 2=$ 49,931; $\mathrm{p}=.187$ ).

Afin de contrôler un éventuel effet d'ordre entre drogue et cannabis, nous avons une fois sur deux intervertit l'ordre de présentation des termes (drogue puis cannabis suivi de cannabis puis drogue). II s'agissait de vérifier que la présentation d'un terme n'active pas d'univers représentation-

(8) II s'agit de produire une série de mot de façon spontanée, à partir d'un mot inducteur, dans le cas présent les mots drogue et cannabis.

(9) Ce mode d'échantillonnage, non représentatif, vise à construire un échantillon afin d'étudier les effets de variables (dans le cas présent le sexe et le type de rapport au cannabis) dans le cadre d'une problématique, les individus sont recrutés de manière semblable à un échantillon par quotas, sur la base des critères retenus.

(10) $\mathrm{Khl} 2=, 000 ; p=1.000$.

(11) $\mathrm{Khl} 2=100,215 ; p=.110$. nels différents, par effet de contexte, lors de la production des évocations du second terme.

Les données issues des entretiens ont fait l'objet d'une analyse par le logiciel Alceste ${ }^{\odot}$ qui permet l'analyse de données textuelles. L'hypothèse générale développée dans le logiciel Alceste ${ }^{\circledR}$ consiste à considérer les lois de distribution du vocabulaire dans les énoncés d'un corpus comme une trace linguistique d'un travail cognitif de reconstruction d'un objet par un individu. II étudie la distribution du vocabulaire (mots pleins) dans les énoncés du corpus (uce) [31]. Alceste ${ }^{\odot}$ regroupe les énoncés en classe d'énoncés ou classe de discours, les énoncés d'une même classe pouvant être définis comme ceux qui possèdent le vocabulaire le plus proche. Le coefficient d'association d'un énoncé à une classe et calculé à partir du Khi 2 d'association, à un degré de liberté, calculé sur le tableau de contingence croisant la présence ou l'absence du mot dans une uce et l'appartenance ou non de cette uce à la classe considérée [29, 30].

Les données issues des questionnaires ont été analysées avec le logiciel EVOC $2000^{\odot}$. Sur la base d'une analyse lexicographique, ce logiciel met en évidence le contenu d'une représentation, et dégage les éléments organisateurs de ce contenu sur la base du croisement de deux critères : la fréquence d'apparition du terme et son rang moyen d'apparition [35], les termes évoqués par les individus seront dès lors plus ou moins saillants sur la base de ce double critère.

\section{Résultats}

Nous présenterons dans un premier temps les données concernant la représentation de la drogue, à travers 
Tableau I: Classes de discours issues de l'analyse Alceste $^{\odot}(n=50)$

\begin{tabular}{|c|c|c|}
\hline $\begin{array}{c}\text { Classe } 1 \\
24,12 \% \text { du discours } \\
18-25 \text { ans Khi } 2=233,95 \\
\text { Femme Khi } 2=7,91\end{array}$ & $\begin{array}{c}\text { Classe } 2 \\
51,48 \% \text { du discours } \\
45-65 \text { ans Khi } 2=222,58 \\
\text { Femme Khi } 2=3,45\end{array}$ & $\begin{array}{c}\text { Classe } 3 \\
24,40 \% \text { du discours } \\
18-25 \text { ans Khi } 2=133,56 \\
\text { Homme Khi } 2=24,63\end{array}$ \\
\hline $\begin{array}{c}\text { La dépendance } \\
\text { Dépendance, dépendant, } \\
\text { besoin, manque, } \\
\text { accoutumance, habitude } \\
\text { Ses effets } \\
\text { Sensation, ressentir, sentir, } \\
\text { envie, bien-être, modifier } \\
\text { Ses conséquences } \\
\text { Crever, souffrant, déchéance, } \\
\text { destruction } \\
\text { Alimentation et dépendance } \\
\text { Chocolat, nourriture, manger }\end{array}$ & $\begin{array}{c}\text { L'argent } \\
\text { Argent, vendre, payer, acheter, } \\
\text { coût, cher, dealer, économie } \\
\text { La famille } \\
\text { Famille, parents, fille, fils, } \\
\text { mère, père } \\
\text { La génération } \\
\text { Jeune, enfant, génération, } \\
\text { époque, âge } \\
\text { La délinquance } \\
\text { Voler, délinquant, tuer... }\end{array}$ & $\begin{array}{c}\text { Les produits } \\
\text { Héroïne, cocaïne, tabac, } \\
\text { cannabis,alcool, ecstasy, Isd } \\
\text { La loi } \\
\text { Légal, légaliser, illégal, hors la loi } \\
\text { réglementer, autoriser } \\
\text { Le cannabis } \\
\text { et son usage } \\
\text { Cannabis, shit, marijuana, } \\
\text { haschich, joints, bambou } \\
\text { La distinction « drogue douce / } \\
\text { drogue dure» }\end{array}$ \\
\hline
\end{tabular}

l'analyse des entretiens suivie de l'analyse des évocations. Dans un deuxième temps nous présenterons les données concernant les représentations du cannabis par l'analyse des entretiens et évocations.

\section{Représentations de la drogue}

\section{Analyse des entretiens}

L'analyse thématique des entretiens permet de souligner l'importance du thème de la dépendance dans l'appréhension de l'objet drogue, $80 \%$ des interviewés abordent spontanément ce thème dans leurs discours, dont $100 \%$ des $18-25$ ans.

L'analyse du corpus à l'aide du logiciel Alceste ${ }^{\odot}$ permet de mettre en évidence trois univers différents de reconstruction de l'objet drogue, trois classes discursives associées à des caractéristiques spécifiques des sujets (tableau I). Le profil de chaque classe est déterminé par l'ensemble des termes et variables (dans le cas présent le sexe et l'âge) les plus significativement présents dans celle-ci. La classe 1 représente $24,12 \%$ du discours total, elle est principalement marquée par le discours des personnes de 18 à 25 ans (Khi $2^{(12)}$ : $233,95)$ et de sexe féminin (Khi 2 : $7,91)$; la classe $2(51,48 \%$ du discours) est elle marquée par le discours des personnes de 45-65 ans (Khi 2 : $222,58)$ et de sexe féminin $(3,45)$; enfin la classe 3 (24,40 \% du discours) est marquée par les propos des personnes de 18-25 ans (Khi $2: 133,56$ ) et de sexe masculin (Khi $2: 24,63$ ).

Une analyse plus approfondie permet de regrouper les termes présents dans chaque classe de discours sous forme de thèmes plus larges.

Pour les femmes de 18-25 ans, le rapport aux drogues se focalise davantage sur la question de la dépendance, aux niveaux physique et psychique, et ses conséquences, souvent appréhendées comme néfastes pour l'individu. Dans le même temps, les effets des substances sur la perception et les sensations interrogent l'enjeu de la consommation et de ses bénéfices éventuels. À noter, la place particulière

(12) Plus le Khi 2 est important, plus la classe de discours est marquée par la variable (sexe et/ou âge). 
qu'occupe l'univers de l'alimentation en tant que champ d'expérience qui sert à penser le processus de dépendance et ses effets dans la reconstruction de l'objet drogue pour cette souspopulation de jeunes femmes.

Pour les personnes plus âgées, l'objet est reconstruit à travers le filtre des générations, la drogue apparaît comme un problème lié à la jeunesse. Le thème de l'argent lié aux échanges marchands de la drogue occupe une place centrale dans les discours des 45-65 ans. Ils l'associent étroitement à d'autres problèmes sociaux, et plus particulièrement à la délinquance. La confrontation à la drogue est posée en tant qu'enjeu dans le cadre de l'univers familial et des relations parentsenfants.

Les discours des jeunes hommes se distinguent nettement, car ils portent plus directement sur les produits et leurs usages. La nomination d'un grand nombre de produits illustre une plus grande proximité fonctionnelle. Elle se traduit par des distinctions entre les substances en fonction des propriétés et de la dangerosité perçues (douce/dure) ou de leur statut légal (alcool, tabac) et par des récits d'expériences directes avec les produits. La place accordée au cannabis et à ses usages dans les discours constitue un des éléments spécifiques de cette sous-population. Les risques engendrés par l'usage sur les plans sanitaires et sociaux sont au cœur des préoccupations exprimées.

Ces résultats montrent qu'en fonction du sexe, mais surtout de l'âge, la drogue n'est pas appréhendée de manière identique.

\section{Analyse des évocations}

L'analyse des évocations effectuées à partir du mot drogue (tableau II), confirme le fait que la notion de dépendance caractérise le mieux la drogue pour les personnes interrogées, le terme dépendance étant le plus saillant parmi ceux cités.

Par ailleurs, les résultats mettent en évidence l'influence des pratiques (consommation de cannabis), sur la construction représentationnelle de la drogue. Le terme mort est plus saillant chez les non-consommateurs, de même que les termes maladie, sida et déchéance leur sont plus caractéristiques. Si le terme mort est présent

Tableau II. Représentations de la drogue selon les modalités de consommation de cannabis

\begin{tabular}{|c|c|c|c|}
\hline & $\begin{array}{c}\text { Non } \\
\text { consommateurs } \\
\text { de cannabis } \\
\text { (n= 100) }\end{array}$ & $\begin{array}{c}\text { Anciens } \\
\text { consommateurs } \\
\text { de cannabis } \\
\text { (n=100) }\end{array}$ & $\begin{array}{c}\text { Consommateurs } \\
\text { actuels de cannabis } \\
\text { (n = 100) }\end{array}$ \\
\hline $\begin{array}{c}\text { Terme } \\
\text { le plus saillant }\end{array}$ & DÉPENDANCE (46) & DÉPENDANCE (49) & DÉPENDANCE (42) \\
\hline Termes saillants & $\begin{array}{c}\text { Mort (31) } \\
\text { Cocaïne (18) } \\
\text { Cérnaïne (18) } \\
\text { Seringue (15) }\end{array}$ & $\begin{array}{c}\text { Cocaïne (21) } \\
\text { Mort (19) } \\
\text { Danger (17) } \\
\text { Héroïne (17) } \\
\text { Dure (16) } \\
\text { Alcool (15) }\end{array}$ & $\begin{array}{c}\text { Cocaïne (22) } \\
\text { Alcool (18) } \\
\text { Héroïne (17) } \\
\text { Danger (15) }\end{array}$ \\
\hline $\begin{array}{c}\text { Termes } \\
\text { relativement } \\
\text { saillants }\end{array}$ & $\begin{array}{c}\text { Maladie (14) } \\
\text { Sida (14) }\end{array}$ & $\begin{array}{c}\text { Seringue (13) } \\
\text { Cannabis (9) } \\
\text { Déchéance (13) }\end{array}$ & $\begin{array}{c}\text { Mort (14) } \\
\text { Cannabis (12) } \\
\text { Ecstasy (10) }\end{array}$ \\
\hline
\end{tabular}


chez les anciens et actuels consommateurs, il n'a pas le même statut.

Enfin, le terme alcool est plus saillant dans la représentation de la drogue chez les ex et actuels consommateurs de cannabis, que chez les nonconsommateurs ; contrairement au terme cannabis, plus saillant chez ces derniers. En dehors de substances, comme la cocaïne et l'héroïne, qui restent emblématiques de l'univers de la drogue, on constate que des mouvements s'opèrent, par le biais des pratiques, quant à l'inscription plus ou moins importante de certaines substances dans l'univers de la drogue.

\section{Représentations du cannabis}

\section{Analyse des entretiens}

L'analyse des discours produits sur le cannabis par les 50 personnes interviewées met en évidence une perception différenciée de cette substance. Pour les personnes les plus âgées (45 ans et plus) le cannabis est perçu comme une drogue, dont l'usage dangereux est associé aux «jeunes". Pour les plus jeunes, le discours sur la dangerosité est plus nuancé. En effet, pour ces derniers on observe une logique de conditionnalité, liée aux modalités de consommation de cannabis. Ce n'est plus la seule consommation de cannabis qui fait du consommateur un drogué, mais les modes (fréquence, consommation matinale, avant le travail) et les contextes (seul versus en groupe) de consommation. Les modalités de consommation de cannabis déterminent par conséquent la signification que peut prendre cette conduite (être ou ne pas être un drogué). Pour ces jeunes, ce ne sont donc pas les propriétés pharmacologiques de la substance qui la définissent comme une drogue, mais les modalités de son utilisation. Au-delà de la dimension intergénérationnelle, c'est la proximité au cannabis, qui conditionne le discours sur cette substance, les jeunes étant plus proches (consommation personnelle, connaissance de consommateurs).

\section{Analyse des évocations}

Les représentations élaborées à propos du cannabis sont influencées par les modalités de consommation de cette substance (tableau III), le terme drogue qui est le terme évoqué le plus saillant pour les non-consom-

Tableau III. Représentations du cannabis selon les modalités de consommation de cannabis

\begin{tabular}{|c|c|c|c|}
\hline & $\begin{array}{c}\text { Non } \\
\text { consommateurs } \\
\text { de cannabis } \\
(\mathbf{n = 1 0 0 )}\end{array}$ & $\begin{array}{c}\text { Anciens } \\
\text { consommateurs } \\
\text { de cannabis } \\
(\mathbf{n = 1 0 0 )}\end{array}$ & $\begin{array}{c}\text { Consommateurs } \\
\text { actuels de cannabis } \\
\text { (n= 100) }\end{array}$ \\
\hline $\begin{array}{c}\text { Terme } \\
\text { le plus saillant }\end{array}$ & DROGUE (64) & JOINT (37) & JOINT (35) \\
\hline Termes saillants & $\begin{array}{c}\text { Joint (32) } \\
\text { Herbe (22) } \\
\text { Plante (22) } \\
\text { Fumer (22) } \\
\text { Feuille (22) } \\
\text { Dépendance (21) }\end{array}$ & $\begin{array}{c}\text { Drogue (33) } \\
\text { Herbe (30) }\end{array}$ & $\begin{array}{c}\text { Herbe (30) } \\
\text { Fumer (23) }\end{array}$ \\
\hline $\begin{array}{c}\text { Termes } \\
\text { relativement } \\
\text { saillants }\end{array}$ & $\begin{array}{c}\text { Shit (10) } \\
\text { Reggae (8) }\end{array}$ & $\begin{array}{c}\text { Feuille (16) } \\
\text { Fumer (15) } \\
\text { Shit (13) } \\
\text { Plante (12) }\end{array}$ & $\begin{array}{c}\text { Drogue (16) } \\
\text { Détente (14) } \\
\text { Plaisir (12) }\end{array}$ \\
\hline
\end{tabular}


mateurs (64/100) ne possède pas le même statut chez les anciens consommateurs $(33 / 100)$, ni chez les actuels consommateurs (16/100), cette variabilité dans la saillance de ce terme témoigne de l'inscription plus (les non-consommateurs) ou moins (les consommateurs) forte du cannabis dans l'univers drogue. La présence du terme dépendance chez les nonconsommateurs et son absence chez les consommateurs et ex-consommateurs renforcent ce constat. Pour les consommateurs actuels, les éléments les plus saillants sont essentiellement liés aux différentes formes et usages de la substance, avec des termes tels que joint, herbe et fumer.

Pour les consommateurs actuels de cannabis, le terme drogue est relativement saillant, au même titre que certains termes qui dénotent l'attribution de propriétés positives à la substance : la détente et le plaisir.

On a donc d'une part une représentation du cannabis qui s'inscrit fortement dans l'univers de la drogue (pour les non-consommateurs) et d'autre part un discours centré sur la substance, les modalités de sa consommation et ses effets (pour les consommateurs actuels). La vision des ex-consommateurs se rapproche de celle des consommateurs actuels.

Ces résultats illustrent les effets des pratiques (actuelles ou passées), qui font que le cannabis n'est pas perçu de façon identique en fonction des relations (usage, ex-usage, non-usage) que les individus entretiennent avec la substance, et mettent en jeu son positionnement dans l'univers des drogues.

\section{Discussion}

Les démarches de prévention reflètent des valeurs que l'on souhaite promouvoir [25]. Ces valeurs propres à la société à un moment donné se dotent d'une apparence de consensus, mais la réalité est toute autre. Comme nous le montrent les résultats de ces recherches sur la drogue et le cannabis, les images et les valeurs sont multiples, déterminées pour partie par les insertions sociales des individus (sexe et âge) et les rapports qu'ils entretiennent avec les substances (consommation versus non-consommation). La pluralité des perceptions de la drogue oriente la catégorisation, les préoccupations et centres d'intérêts de ces derniers à travers la valorisation de certaines thématiques.

Ces résultats mettent à jour un double effet des pratiques de consommation de cannabis sur la construction représentationnelle liée à l'objet drogue. Premièrement, on peut constater une image plus négative de l'objet drogue chez les non-consommateurs de cannabis. Les consommateurs de cannabis, quant à eux, incluent davantage l'alcool dans l'univers de la drogue. S'agit-il dès lors, d'une attitude «défensive » pour atténuer le marquage social «négatif » de la consommation de cannabis en incluant l'alcool dans l'univers des drogues, ou l'expression de l'intégration des nouveaux discours de prévention (inclusion des substances psychoactives en dehors de leurs statuts légaux).

La logique de conditionnalité mis en évidence dans la définition du "drogué au cannabis " met à jour quant à elle des raisonnements qui puisent dans les psychologies naïves et les théories de sens commun [15]. Ces types de raisonnements ont par ailleurs déjà été mis en évidence au sujet de l'alcool, le «bien-boire» apparaissant comme une norme de conduite à laquelle déroge la personne qui boit trop et/ou seule; passant ainsi du statut de simple consom- 
mateur d'alcool à celui d'alcoolique [6, $8,9,12]$.

Les pratiques déclarées par les individus influencent donc les représentations que ces derniers développent vis-à-vis de la drogue mais aussi du cannabis, mettant à jour des enjeux différents en termes de communication préventive.

Certaines études soulignent le rôle important joué par les perceptions des risques sanitaires et de la désapprobation sociale dans l'évolution de la prévalence [4, 24]. Pour les consommateurs de cannabis, que nous avons interrogés, la référence à l'univers de la drogue est moindre dans leur représentation de cette substance. Le cannabis est perçu sous un angle de proximité instrumentale et investi de significations hédonistes, quant aux références à la dangerosité de la substance, elles sont absentes. La substance n'est plus appréhendée comme c'est le cas pour les non-consommateurs principalement comme une drogue, elle gagne de fait en "acceptabilité sociale", sa nocivité n'apparaît plus de même dans la définition de l'objet.

Ce résultat invite à réfléchir quant à l'opportunité de penser une prévention spécifique visant la réduction des risques encourus dans certaines formes de relation à cette substance, au regard de l'étendue de son usage chez les jeunes adultes, et ce notamment en prenant en compte les compétences [33] et savoirs des publics visés.

Depuis notamment le rapport Roques [32], l'état des savoirs scientifiques sur la dangerosité des drogues a permis de repenser les risques sanitaires et les démarches de prévention associés à certaines substances, suscitant de fait, un débat vivace emprunt de stéréotypes et de clivages idéologiques. Comme nous l'avons vu à travers les résultats de ces recherches, les consommateurs de cannabis associent davantage que les nonconsommateurs, l'alcool à l'univers de la drogue, ce résultat est à mettre en perspective avec la tendance observée chez les usagers de cannabis à estimer souvent, les dangers de ce produit, en prenant pour point de comparaison l'alcool supposé engendrer une dépendance et une morbidité incomparable $[3,16]$. Ce phénomène illustre les effets que peuvent avoir les pratiques de consommation sur le jeu de classification des substances, et notamment le positionnement de l'alcool [5]. Dans le contexte actuel de redéfinition des risques liés aux substances psychoactives, ces constats invitent à s'interroger sur la façon dont sont décodées et instrumentalisées les nouvelles campagnes d'information en fonction des pratiques antérieures des individus et des groupes. Comme nous le montrent ces résultats, la diffusion des messages de prévention se heurte aux filtres que représentent les pratiques de consommation des individus et des groupes, qui régulent l'information afin de l'accorder avec les systèmes de représentations préexistants.

\section{Conclusion}

L'ensemble des résultats présentés souligne le caractère heuristique de la théorie des représentations sociales dans l'étude de la dynamique des perceptions des substances psychoactives et dans la réflexion pour la prévention. Ces résultats montrent bien que l'étude des représentations sociales peut être un outil au service de la prévention [1] qui permet à la fois d'appréhender les aspects psychosociaux en jeu dans la réappropriation des nouvelles connaissances et de cibler des pistes d'intervention en fonction des situations spécifiques. 


\section{BIBLIOGRAPHIE}

1. Abric JC. Exclusion, insertion et prévention. Toulouse : Erès, $1996: 168 \mathrm{p}$.

2. Abric JC. Pratiques sociales et représentations. Paris : Presses Universitaires de France, 1994 : $252 \mathrm{p}$.

3. Aquatias S, Maillard I, Zorman M. Faut-il avoir peur du haschisch? Entre diabolisation et banalisation : les vrais dangers pour les jeunes. Paris : Syros, $1999: 224$ p.

4. Bachman JG, Johnston LD, O'Malley PM. Explaining recent increase in students' marijuana use : impact of perceived risks and disapproval, 1976 through 1996. Am J Public Health 1998; 88 (6) : 887-92.

5. Beck F, Peretti-Watel P. EROPP99 : enquête sur les représentations, opinions et perceptions relatives aux psychotropes. Paris : OFDT, $2000: 203$ p.

6. Bernand C. Désirs d'ivresse : Alcools, rites et dérives. Paris : Autrement $2000: 194$ p.

7. Boissonnat V. Représentation sociale de l'alcool dans la bande dessiné. Haut Comité d'Étude et d'Information sur l'Alcoolisme, $1988: 181 \mathrm{p}$.

8. Cerclé A. L'alcoolisme. Paris : Flammarion, $1998: 127 \mathrm{p}$.

9. Chapuis R. L'alcool, un mode d'adaptation sociale. Paris : L'Harmattan, 1989 : 192 p.

10. Chauchat H. L'enquête en psycho-sociologie. Paris : PUF, $1985: 253$ p.

11. Derrida J. Rhétorique de la drogue. In : Hervieu J-M. L'esprit des drogues. Paris : Autrement, 1993 : $285 \mathrm{p}$.

12. Dubet F. Les deux drogues. In : Ehrenberg A, Mignon P. Drogues, politique et société. Paris : Le Monde, 1992 : $369 \mathrm{p}$.

13. Fatela J. Drogues et ambivalences de la subjectivité. In : Ehrenberg A. Individus sous influence. Paris : Esprit, 1991 : 355 p.

14. Guilbert A, Baudier F, Gautier A. Baromètre Santé 2000. Vanves : CFES, $2001: 473$ p.

15. Heider F. The Psychology of Interpersonal Relations. New-York : John Wiley \& Sons, $1958: 322 \mathrm{p}$.

16. Ingold R, Toussirt M. Le cannabis en France. Paris : Anthropos, $1998: 191 \mathrm{p}$.

17. INSERM. Cannabis : Quels effets sur la santé ?. Paris : INSERM, $2001: 375 \mathrm{p}$.

18. Jodelet $\mathrm{D}$. Représentation sociale : phénomène, concept et théorie. In : Moscovici S. Psychologie sociale. Paris : PUF, 1984 : 575 p.

19. Jodelet D. Les représentations sociales. Paris : PUF, $1989: 456 \mathrm{p}$.

20. Lefèvre F, Simioni AM. Marijuana, health, disease, and freedom : analysis of an internet forum. Cadernos de Saude Publica 1999;15,161-8.

21. Morin M, Apostolidis T. Contexte social et santé. In : Fischer GN. Traité de psychologie de la santé. Paris : Dunod, 2002.

22. OFDT (Observatoire Français des Drogues et des Toxicomanies). Drogues et toxicomanies : indicateurs et tendances. Paris : OFDT, $1999: 270 \mathrm{p}$.

23. OFDT (Observatoire Français des Drogues et des Toxicomanies). Drogues et dépendances : indicateurs et tendances. Paris : OFDT, $2002: 368 \mathrm{p}$.

24. Pacula RL, Grossman M, Chaloupka FJ, O'Malley PM, Johnston LD, Farelly MC. Marijuana and youth. In : Risky Behavior among Youths : An Economic Analysis. Chicago : The University of Chicago Press, 2001 : chap 6.

25. Parquet PJ. Pour une prévention de l'usage des substances psychoactives. Paris : CFES, 1998 : $46 \mathrm{p}$.

26. Pélicier Y, Thuillier G. La drogue. Paris : PUF, 1972 : 127 p.

27. Peretti-Watel P. Comment devient-on fumeur de cannabis? Une perspective quantitative. $\mathrm{Rev} F r$ Sociol 2001; 42 (1) : 3-27.

28. Perrin M, Anthropos. In : Hervieu JM. L'esprit des drogues. Paris : Autrement, $1993: 285$ p.

29. Reinert M. Quelques interrogations à propos de l'«objet» d'une analyse de discours de type statistique et de la réponse «Alceste». Langage et Societe 1999; $90: 57-70$.

30. Reinert M. Alceste, une méthodologie d'analyse des données textuelles et une application : Aurélia de Gérard de Nerval. Bull Methodol sociol 1990; $26: 24-54$.

31. Reinert M. Un logiciel d'analyse lexicale (ALCESTE). Cah Anal Donnees 1986; 4 : 471-84

32. Roques B. La dangerosité des drogues. Paris : Odile Jacob 1999 : 318 p.

33. Rosenbaum M. «Just say known » to teen-agers and marijuana. J Psychoactive Drugs 1998 ; 30 (2) : 197-203.

34. Thrush D, Fife-Schaw C, Breakwell GM. Young people's representations of others' views of smoking : is there a link with smoking behaviour? J Adolesc $1997 ; 20,57-70$.

35. Verges P. Approche du noyau central : propriétés quantitatives et structurales. In : Guimelli C. Structures et transformations des représentations sociales. Lausanne : Delachaux et Niestlé, 1994 $277 \mathrm{p}$. 